\title{
Allocating Bandwidth based on Users' Web Usage Patterns for University Networks: A Hybrid Data Mining Approach
}

\author{
Rolysent K. Paredes ${ }^{1}$, Ariel M. Sison ${ }^{2}$, and Ruji P. Medina ${ }^{3}$
}

\begin{abstract}
Due to the limited bandwidth and continuous growth of users make the IT managers focus on the strategies concerning bandwidth allocation. Thus, managing the bandwidth in campus networks has become one of the challenges in recent years. This paper introduces a mechanism for bandwidth allocation based on the users' web usage patterns. The main purpose is to set a higher bandwidth to the users who are inclined to browsing educational websites compared to those who are not. In attaining this proposed technique, a hybrid data mining approach were done which composed of preprocessing of the weblogs, class labeling of the dataset, computation of the feature subspaces, training for the development of the ANN for LDA/GSVD algorithm, and visualization. The proposed method was applied to real weblogs from university's proxy servers which include 104 active users. The results indicate that the proposed method is useful in classifying those users who used the internet in an educational way and those who are not. Thus, the developed ANN for LDA/GSVD algorithm outperformed the existing algorithm up to $50 \%$ which indicates that this approach is efficient. Based on the results, $35.06 \%$ of the users browsed educational contents. Through this technique, users will be encouraged by using the internet for educational purposes. Moreover, IT managers can make better decisions to optimize the allocation of bandwidth resources.
\end{abstract}

Keywords - Bandwidth Allocation, Linear Discriminant Analysis, Neural Network for LDA/GSVD, Web Usage Patterns s.

\section{INTRODUCTION}

One of the essential challenges in managing multi-service computer networks such as university networks is to control the bandwidth efficiently which plays a critical role in many internet services [1]. Thus, allocating bandwidth in a fair and efficient way is a central issue in networking due to its limitation [2] and because of that many scientists have focused on fairness goals in bandwidth management [1]. Also, bandwidth allocation is crucial in network design, deployment, and maintenance [3].

Due to the importance of bandwidth allocation, this study aims to provide a new mechanism for allocating bandwidth to network users by utilizing the weblogs that are stored in the

Rolysent K. Paredes ${ }^{1},{ }^{1}$ Technological Institute of the Philippines, Quezon City, Philippines

Ariel M. Sison ${ }^{2},{ }^{2}$ Emilio Aguinaldo College, Manila, Philippine

Ruji P. Medina ${ }^{3},{ }^{3}$ Technological Institute of the Philippines, Quezon City, Philippines. proxy servers [4]. These weblogs contain latent information of the network users that can be used to discover user patterns through the use of data mining techniques such as classification which includes C4.5, ID3, K-Nearest Neighbor (KNN) classifier, Naive Bayes, Support Vector Machine (SVM), Artificial Neural Network (ANN), and Linear Discriminant Analysis (LDA) [5-7].

To discover users' web usage patterns from the weblogs, LDA was used since it is the most reliable trained classification model compare to the others and has been used extensively in the past decades for dimensionality reduction, recognition, and supervised classification [5]. Further, LDA has the advantage in seeking projection vector that yields optimal discrimination between distinct groups of observations [8]. However, it cannot deal with unlabeled datasets $[9,10]$ and fails when there exists a singularity of matrix due to small sample size (SSS) problem [11-13]. Thus, this study aims as well to address the two issues of LDA before it is applied to the weblogs to identify patterns. Typically weblogs are unlabeled, and due to the dynamic data that composes it, SSS problem can occur.

There were several ways to manage the unlabeled dataset most common method approach is making LDA unsupervised by utilizing clustering algorithms [9, 14-16]. With that, SelfOrganizing Map (SOM) was employed in creating the clusters or making of the class labels because it is an unsupervised learning technique to discover patterns to the dataset and it can deal with high dimensional data [17]. It is also an example of an artificial neural network architecture which makes it fast and robust $[18,19]$.

In dealing with SSS problem in LDA, several solutions have been proposed such as in the studies of [13, 20-23]. But the most common approach is the application of Generalized Singular Value Decomposition on LDA (GSVD) [24] which is widely used by various discriminant analysis techniques [25]. It is also the most common method for solving the matrix singular problems in many mathematical computations $[25$, 26]. Further, GSVD in LDA (LDA/GSVD) provides high recognition accuracy that is why many researchers used and developed variance of it [27]. However, GSVD suffers from computational cost which can cause a longer time in classifying datasets when applied to LDA [27-29]. Moreover, if there is a new instance for classification in the existing 
LDA/GSVD, the whole process of the algorithm will be repeated from the very start.

In solving the problems of the existing LDA/GSVD, the Artificial Neural Network (ANN) algorithm was developed. The ANN algorithm eliminates the mathematical computations and numerous iterations that are involved in the existing LDA/GSVD algorithm which compromise time complexity making it less efficient. Further, with this method, learning can be done from the weblog and classification to each instance, whether new or previously part of the training or testing, will be faster because it will not go back to the start of the whole procedure. The use of ANN in developing the algorithm has the advantage of accuracy because it provides a faster response. Also, ANN was equipped with the distinctiveness of parallel processing, non-linear mapping, associative memory and offline/online learning abilities [30, 31].

Hence, the primary purpose of the study is to introduce a new mechanism for allocating bandwidth to the users which can be used mainly on the university's networks. This proposed bandwidth allocation mechanism can help the network administrator or those who manage the network in school in identifying the users who educationally utilized the internet. This approach can also assist them in their decision and policy-making concerning the utilization of the internet. This new technique utilized the weblogs from the proxy server which was used for discovering the users' web usage patterns through the use of ANN for unsupervised LDA/GSVD. The idea is that users will be assigned with higher bandwidth if they are classified or inclined in accessing more on educational websites; otherwise, they will have a lower bandwidth. With this approach, it encourages the users in the campus to engage more in accessing educational contents, be focused in their studies especially the students, and the faculty and staff will be more active in research and development. For LDA to work efficiently in determining users who browsed more educational websites or not, SOM was used for class labeling, and ANN for LDA/GSVD was employed for faster classification and computation of the feature subspaces. These feature subspaces separate well the users according to their web usage patterns. The results of this study will be an excellent contribution to the continuous development in the area of computer networking.

\section{II.METHODS}

In attaining this new bandwidth allocation approach, the ANN for LDA/GSVD algorithm must be developed first because it will be used in discovering users' web usage patterns. Thus, this algorithm will be used to visualize appropriately the groups of users who are inclined to accessing educational sites and those who are not. The process of developing algorithm is presented in figure 1 which shows four (4) major stages these are preprocessing, class labeling, computation of feature subspaces, and ANN training.

\section{A. Preprocessing}

In this step, the unnecessary instances from the weblogs will be removed. This also includes the identification of the category of the website and who requests for it. There are four (4) objects involve in preprocessing these are the weblog, captive portal log, shallalist files, and the predefined educational sites. The weblog and captive portal log were taken from the proxy servers in Misamis University, Ozamiz City, Philippines. It is composed of the logs in the 1st semester of 2017-2018. It mainly contains the websites accessed by the wired and wireless users while the captive portal log includes the date and time when the users login or use the internet. This captive portal $\log$ [32] will be used to identify the specific user who requests the particular website. The shallalist file contains the category of websites which will be utilized in determining where the requested website belongs [33, 34]. Lastly, the predefined educational sites is a file that contains lists of educational websites which will prevail even though they are differently categorized in the shallalist. Table 1 presents the algorithm for the preprocessing.

\section{TABLE I: PREPROCESSING ALGORITHM}

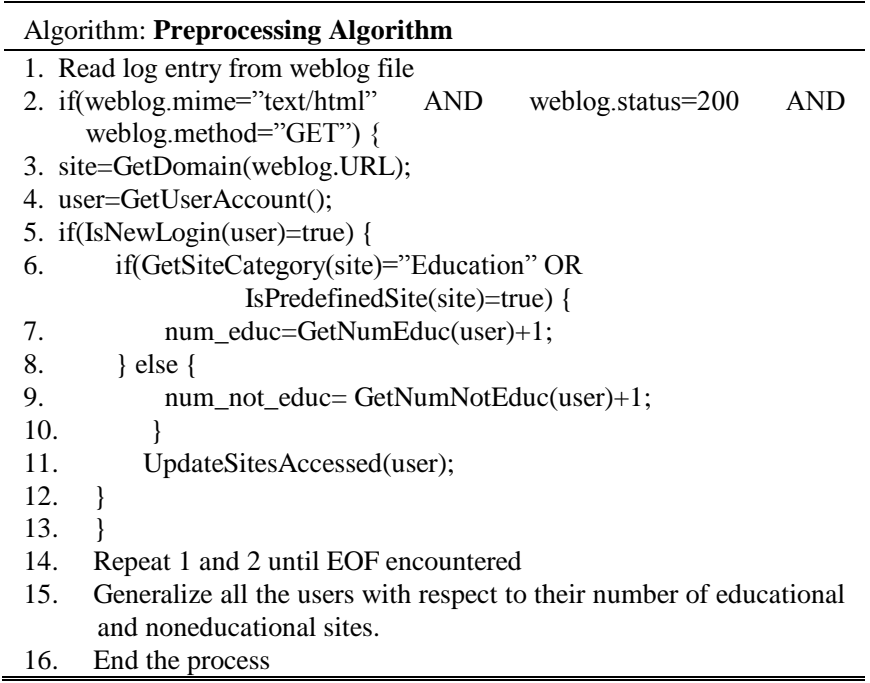

\section{B. Class Labelling}

After the preprocessing, the generalized data is unlabeled and because of that class labeling is imperative for the LDA/GSVD to work. SOM is employed to create these clusters which become the class labels.

\section{Computation of Feature Subspaces}

At this point, the dataset has been labeled and ready for actual computation of the feature subspaces. For the ANN architecture to learn, predict, and classify the users' web usage patterns, the feature subspaces must be derived from that existing LDA/GSVD algorithm. These feature subspaces are actual points where the users belong, and these are used for further visualization of the classification of the users. Thus, it is the current algorithm that is used so that the accuracy of the developed ANN algorithm will be the same.

\section{D.ANN Training}

The tansigmoid transmission function was used as the activation function in the hidden layers. The network is formed from 2 input variables which are the dimensions or the 
frequency of the educational websites and noneducational websites per user, and the corresponding three output variables which are the expected feature subspaces and class label. These dimensions and feature subspaces will be used in training and testing. As for the number of hidden layer nodes, $70 \%$ of the input values were allocated for training samples, and $30 \%$ for the testing. Moreover, Bayesian Regularization Back Propagation (BRBP) $[35,36]$ was employed to train the network.

After saving the trained network, it will become a module or subroutine that will be used to solve the expected new feature subspaces of the inputs. Thus, the algorithm shown in table 2 is for the proposed bandwidth allocation mechanism. Further, the architecture of the trained network which is used to separate the groups of users according to their web usage patterns is presented in figure 2 .

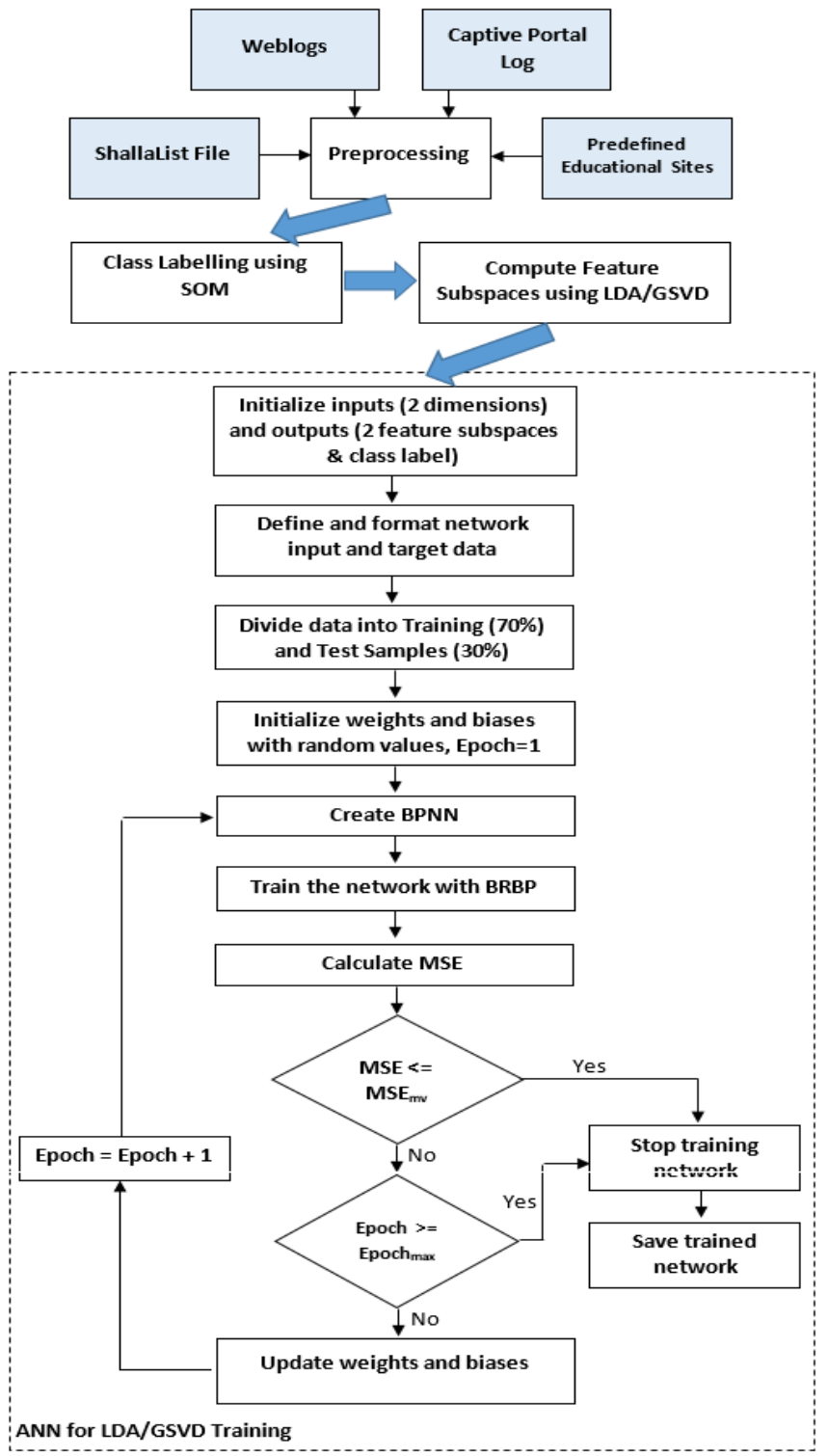

Fig. 1. Training process for the development of the ANN for LDA/GSVD algorithm.
TABLE II: PROPOSED BANDWIDTH ALLOCATION MECHANISM ALGORITHM

Algorithm: Bandwidth Allocation Mechanism Algorithm

1. Open Dataset

2. Get each user's total number of educational and noneducational websites.

3. Compute the corresponding feature subspaces and determine the class label using the ANN for LDA/GSVD.

4. Classify and visualize the user's web usage pattern.

5. Allocating bandwidth to the user.

6. Repeat 1 and 2 until EOF encountered

7. End Process

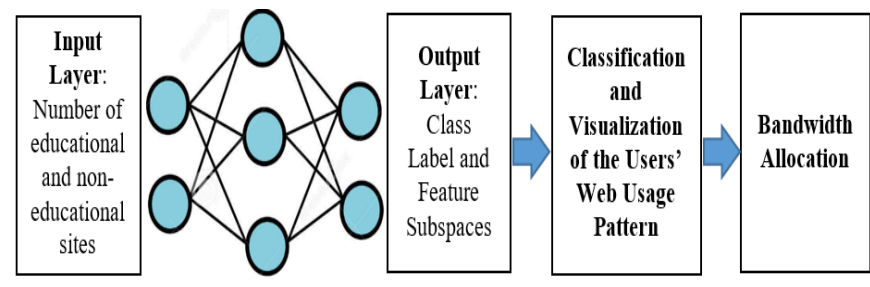

Hidden Layer: 30 hidden neurons

Fig. 2. Architecture of the Trained ANN for LDA/GSVD used in determining Users' Web Usage Pattern and Bandwidth Allocation

As shown in figure 2, the inputs to the trained ANN for GSVD are the dimensions or the total number of educational and noneducational by each user. The trained network will compute the feature subspaces and classify the users' web patterns. In viewing the actual separation of the users according to their patterns, visualization will be presented as well to find out the density of the users who manage to browse educational websites and those who are not. This visualization can be a great help for the network administrators or IT managers in allocating the specific amount of bandwidth.

\section{RESULTS AND DISCUSSIONS}

\section{A. Actual Dataset}

After the preprocessing step, it was identified that there are a total of 104 active users in specific network nodes for the $1 \mathrm{st}$ semester of school year 2017-2018. Therefore, this study used a dataset that has 104 instances and two dimensions. Figure 3 shows the graph that corresponds to the total number of educational websites and noneducational websites that were accessed by the users. Table 3 shows some instances of the dataset. User accounts were replaced with "User 1", "User 2", and etc.

TABLE III: PARTS OF THE DATASET

\begin{tabular}{lll}
\hline \hline Active Users & Educational Websites & $\begin{array}{l}\text { Noneducational } \\
\text { Websites }\end{array}$ \\
\hline User 1 & 243 & 329 \\
User 2 & 154 & 489 \\
User 3 & 283 & 77 \\
User 4 & 204 & 44 \\
User 5 & 54 & 443 \\
\hline
\end{tabular}

After preprocessing, class labeling comes in. Table 4 presents the number of users belongs to the classes. 
TABLE IV: FREQUENCY OF THE CLASSES

\begin{tabular}{ll}
\hline \hline Class Label & Number of Network Users \\
\hline 1 & 27 \\
2 & 77 \\
\hline \hline
\end{tabular}

B. Computation and Visualization of the Feature Subspaces using the Existing LDA/GSVD Algorithm

Once all the instances of the dataset are labeled, the computation of the feature subspaces was done. It took 4 seconds for the existing LDA/GSVD to finish the whole process. Figure 3 shows the graph for the feature subspaces separating the classes. Each point in the graph represents a user. Thus, class 1 composed of fewer users compared to class 2. It is noticeable that all data points are appropriately separated for better visualization.

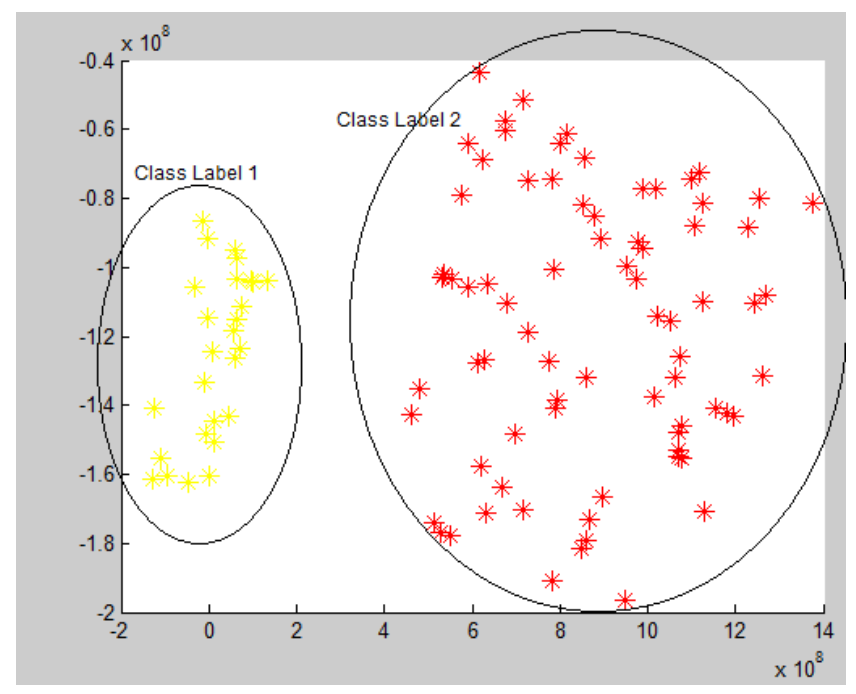

Fig. 3. Graph of the dataset after applying the existing LDA/GSVD

\section{Training for the development of ANN for LDA/GSVD algorithm}

After getting all the necessary values such as class labels and feature subspaces, training will be next. This study used the performance functions, the Mean Squared Error (MSE) and Regression (R), to conduct the performance evaluation of the ANN for LDA/GSVD. MSE is the average squared difference between observed output values from ANN and the targets introduced in the training dataset for the training samples. A small MSE value means that the developed ANN algorithm can obtain insignificant error. Regression (R) calculates the correlation between outputs and targets. When values of $\mathrm{R}$ are equal to 1 , it means a close relationship, and if $\mathrm{R}$ is equal to 0 , there is a random relationship [36]. Thus, table 5 shows that the developed algorithm has high accuracy because of lower MSE values and the value of Regression (R) for the training, and testing is 1 .

TABLE V: PERFORMANCE OF THE ANN FOR LDA/GSVD USING BRBP

\begin{tabular}{lll}
\hline \hline Dataset Sample & MSE & Regression \\
\hline Training & 0.6945 & 1 \\
Testing & 0.7336 & 1 \\
\hline \hline
\end{tabular}

\section{Computation and Visualization of the Feature Subspaces using the ANN for LDA/GSVD Algorithm}

It is noticeable that figure 4 which presents the classification of the data using the enhanced algorithm is very much similar to figure 3 which utilized the existing LDA/GSVD. It is a manifestation that the accuracy of the improved LDA/GSVD maintains the accuracy of current LDA/GSVD algorithm. Thus, if a new user joins the network, this developed algorithm can be used directly in identifying his/her web usage patterns whereas the existing LDA/GSVD, it's the whole algorithm that will be executed and all the instances of the dataset will be considered during the process. With that, the existing algorithm can be exhaustive especially when there is a new instance added to the dataset. The whole process took 2 seconds.

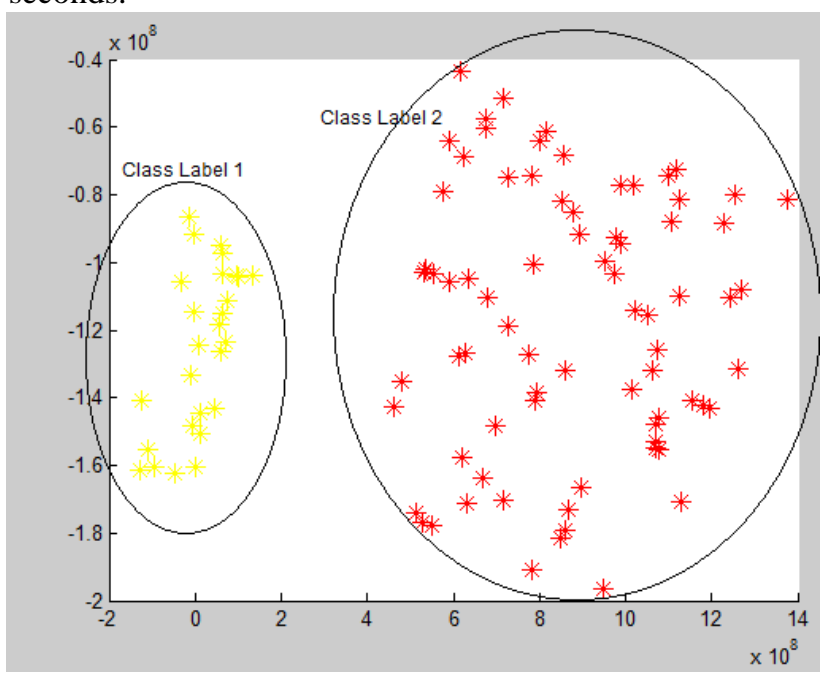

Fig. 4. Graph of the dataset after applying the ANN for LDA/GSVD Algorithm

Table 6 presents the computational costs of two algorithms. It is evident that the enhanced LDA/GSVD improved the computational cost by $50 \%$. The values for the computational costs may be too small because there are only 104 instances that composed the dataset.

TABLE VI: COMPUTATIONAL COSTS OF THE EXISTING AND ENHANCED ALGORITHMS

\begin{tabular}{ll}
\hline \hline Algorithm & $\begin{array}{l}\text { Computational Cost or } \\
\text { Total Execution Time }\end{array}$ \\
\hline Existing LDA/GSVD & 4 seconds \\
ANN for LDA/GSVD & 2 seconds \\
Improvement of the Enhanced LDA/GSVD & $50 \%$ \\
\hline \hline
\end{tabular}

\section{E. Bandwidth Allocation}

Based on the visualization of the feature subspaces, it can be seen that users who belong to class 1 are those individuals who are inclined to doing research or browsing educational websites. There are 27 users belong to class 1 who should be given a higher bandwidth compared to the users in class 2. For this case, the algorithm for assigning bandwidth to each user is presented in table 7; these are the particular lines of instructions to the step 5 of the algorithm shown in table 2 . The IT manager must define the values for the higher and lower bandwidths. 
TABLE VII: ALLOCATING BANDWIDTH TO EACH USER

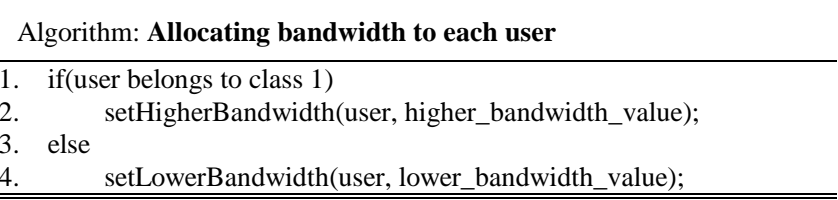

\section{CONCLUSIONS AND RECOMMENDATIONS}

In this study, focusing on the web usage patterns in bandwidth allocation, a hybrid data mining technique based on clustering, ANN, and discriminant analysis was presented and applied on a real weblog of a university network. The main result is that this method is useful in assigning bandwidth to the users. Thus, simulation results showed that discovering of the users' web usage patterns can be done faster using the developed ANN for LDA/GSVD algorithm. Besides, the enhanced algorithm is efficient while maintaining the accuracy of the existing algorithm. Through this proposed approach, network administrators, IT managers, or those who manage the school's network could identify those users that will be allocated with higher bandwidth. With this method, the limited bandwidth in the campus will be appropriately utilized for educational purposes. Further, the results of this study could provide IT managers with useful knowledge to develop their decisions and policies regarding the utilization of the internet especially that there are only $35.06 \%$ of the active users who used the internet in educational purposes.

\section{REFERENCES}

[1] E. A. Z. Noughabi, B. H. Far, and B. Raahemi, "Predicting Students Behavioral Patterns in University Networks for Efficient Bandwidth Allocation: A Hybrid Data Mining Method (Application Paper)," 2016 IEEE 17th International Conference on Information Reuse and Integration (IRI), 2016. https://doi.org/10.1109/IRI.2016.21

[2] A. Sarigiannidis and P. Nicopolitidis, "Addressing the interdependence in providing fair and efficient bandwidth distribution in hybrid opticalwireless networks," International Journal of Communication Systems, vol. 29 , no. 10 , pp. $1658-1682,2015$. https://doi.org/10.1002/dac.3080

[3] M. Hemmati, B. Mccormick, and S. Shirmohammadi, "Fair and Efficient Bandwidth Allocation for Video Flows Using Sigmoidal Programming," 2016 IEEE International Symposium on Multimedia (ISM), 2016 https://doi.org/10.1109/ISM.2016.0052

[4] P. Sukumar, L. Robert, and S. Yuvaraj, "Review on modern Data Preprocessing techniques in Web usage mining (WUM)," 2016 International Conference on Computation System and Information Technology for Sustainable Solutions (CSITSS), 2016. https://doi.org/10.1109/CSITSS.2016.7779441

[5] N. B. M. Zainee and K. Chellappan, "A preliminary dengue fever prediction model based on vital signs and blood profile," 2016 IEEE EMBS Conference on Biomedical Engineering and Sciences (IECBES), 2016 https://doi.org/10.1109/IECBES.2016.7843530

[6] S. G. Langhnoja, M. P. Barot, and D. B. Mehta, "Web usage mining using association rule mining on clustered data for pattern discovery," International Journal of Data Mining Techniques and Applications, vol. 2, no. 1, 2013

[7] S. S. Nikam, "A comparative study of classification techniques in data mining algorithms," Oriental Journal of Computer Science and Technology, vol. 8, no. 1, pp. 13-19, 2015.
[8] S. Yu, Z. Cao, and X. Jiang, "Robust linear discriminant analysis with a Laplacian assumption on projection distribution," 2017 IEEE International Conference on Acoustics, Speech and Signal Processing (ICASSP), 2017. https://doi.org/10.1109/ICASSP.2017.7952620

[9] C. L. Liu, W. H. Hsaio, C. H. Lee, and F. S. Gou, "Semi-Supervised Linear Discriminant Clustering," IEEE Transactions on Cybernetics, vol. 44, no. 7, pp. 989-1000, 2014. https://doi.org/10.1109/TCYB.2013.2278466

[10] P. P. Markopoulos, "Linear Discriminant Analysis with few training data," 2017 IEEE International Conference on Acoustics, Speech and Signal Processing (ICASSP), 2017. https://doi.org/10.1109/ICASSP.2017.7953033

[11] W. Deng, J. Hu, and J. Guo, "Extended SRC: Undersampled Face Recognition via Intraclass Variant Dictionary," IEEE Transactions on Pattern Analysis and Machine Intelligence, vol. 34, no. 9, pp. 18641870, 2012 https://doi.org/10.1109/TPAMI.2012.30

[12] J. Shao, Y. Wang, X. Deng, and S. Wang, "Sparse linear discriminant analysis by thresholding for high dimensional data," The Annals of Statistics, vol. 39, no. 2, pp. 1241-1265, 2011. https://doi.org/10.1214/10-AOS870

[13] Z. Wang, Y.-H. Shao, L. Bai, C.-N. Li, L.-M. Liu, and N.-Y. Deng, "MBLDA: A novel multiple between-class linear discriminant analysis," Information Sciences, vol. 369, pp. 199-220, 2016. https://doi.org/10.1016/j.ins.2016.05.031 https://doi.org/10.1016/j.ins.2015.10.028

[14] C. Ding and T. Li, "Adaptive dimension reduction using discriminant analysis andK-means clustering," Proceedings of the 24th international conference on Machine learning - ICML 07, 2007. https://doi.org/10.1145/1273496.1273562

[15] D. Wang, F. Nie, and H. Huang, "Unsupervised Feature Selection via Unified Trace Ratio Formulation and K-means Clustering (TRACK)," Machine Learning and Knowledge Discovery in Databases Lecture Notes in Computer Science, pp. 306-321, 2014. https://doi.org/10.1007/978-3-662-44845-8_20

[16] D. Conka, P. Viszlay, and J. Juhar, "Improved class definition in two dimensional linear discriminant analysis of speech," 2015 25th International Conference Radioelektronika (RADIOELEKTRONIKA), 2015. https://doi.org/10.1109/RADIOELEK.2015.7129025

[17] J. Faigl and G. A. Hollinger, "Autonomous Data Collection Using a Self-Organizing Map," IEEE Transactions on Neural Networks and Learning Systems, vol. 29, no. 5, pp. 1703-1715, 2018. https://doi.org/10.1109/TNNLS.2017.2678482

[18] R. Mclean, A. J. Walker, and G. Bright, "An artificial neural network driven decision-making system for manufacturing disturbance mitigation in reconfigurable systems," 2017 13th IEEE International Conference on Control \& Automation (ICCA), 2017. https://doi.org/10.1109/ICCA.2017.8003144

[19] S. M. Sharif, F. M. Kusin, Z. H. Asha'Ari, and A. Z. Aris, "Characterization of Water Quality Conditions in the Klang River Basin, Malaysia Using Self Organizing Map and K-means Algorithm," Procedia Environmental Sciences, vol. 30, pp. 73-78, 2015. https://doi.org/10.1016/j.proenv.2015.10.013

[20] ] Z. Zhang, G. Dai, C. Xu, and M. I. Jordan, "Regularized discriminant analysis, ridge regression and beyond," Journal of Machine Learning Research, pp. 2199-2228, Aug. 2010.

[21] T. Zhang, B. Fang, Y. Y. Tang, Z. Shang, and B. Xu, "Generalized Discriminant Analysis: A Matrix Exponential Approach," IEEE Transactions on Systems, Man, and Cybernetics, Part B (Cybernetics), vol. 40, no. 1, pp. 186-197, 2010. https://doi.org/10.1109/TSMCB.2009.2024759

[22] J. Zhao, L. Shi, and J. Zhu, "Two-Stage Regularized Linear Discriminant Analysis for 2-D Data," IEEE Transactions on Neural Networks and Learning Systems, vol. 26, no. 8, pp. 1669-1681, 2015. https://doi.org/10.1109/TNNLS.2014.2350993

[23] J. K. P. Seng and K. L.-M. Ang, "Big Feature Data Analytics: Split and Combine Linear Discriminant Analysis (SC-LDA) for Integration Towards Decision Making Analytics," IEEE Access, vol. 5, pp. 14056$14065,2017$. 
https://doi.org/10.1109/ACCESS.2017.2726543

[24] P. Howland and H. Park, "Generalizing discriminant analysis using the generalized singular value decomposition," IEEE Transactions on Pattern Analysis and Machine Intelligence, vol. 26, no. 8, pp. 9951006, 2004. https://doi.org/10.1109/TPAMI.2004.46

[25] X. Jing, Y. Dong, and Y. Yao, "Uncorrelated optimal discriminant vectors based on generalized singular value decomposition," International Conference on Automatic Control and Artificial Intelligence (ACAI 2012), 2012. https://doi.org/10.1049/cp.2012.1444

[26] Z. Chen and T. H. Chan, "A truncated generalized singular value decomposition algorithm for moving force identification with ill-posed problems," Journal of Sound and Vibration, vol. 401, pp. 297-310, 2017. https://doi.org/10.1016/j.jsv.2017.05.004

[27] W. Wu and M. O. Ahmad, "Orthogonalized linear discriminant analysis based on modified generalized singular value decomposition," 2009 IEEE International Symposium on Circuits and Systems, 2009. https://doi.org/10.1109/ISCAS.2009.5118084

[28] S. Bahrami, "Three-dimensional inverse scattering approach using analytical singular value decomposition method," 2017 18th International Radar Symposium (IRS), 2017. https://doi.org/10.23919/IRS.2017.8008247

[29] H. Zhao and P. C. Yuen, "Incremental Linear Discriminant Analysis for Face Recognition," IEEE Transactions on Systems, Man, and Cybernetics, Part B (Cybernetics), vol. 38, no. 1, pp. 210-221, 2008. https://doi.org/10.1109/TSMCB.2007.908870

[30] Y. Dash and S. K. Dubey, "Quality prediction in object oriented system by using ANN: a brief survey," International Journal of Advanced Research in Computer Science and Software Engineering, vol. 2, no. 2, 2012.

[31] S. S. Ranhotra, A. Kumar, M. Magarini, and A. Mishra, "Performance comparison of blind and non-blind channel equalizers using artificial neural networks," 2017 Ninth International Conference on Ubiquitous and Future Networks (ICUFN), 2017 https://doi.org/10.1109/ICUFN.2017.7993785

[32] F. L. Aryeh, M. Asante, and A. E. Y. Danso, "Securing Wireless Network Using pfSense Captive Portal with Radius Authentication-A Case Study at UMaT," Ghana Journal of Technology, vol. 1, no. 1, pp. 40-45, 2016

[33] Shalla Secure Services KG, "Shalla's Blacklists," Shalla Secure Services, 2018. [Online]. Available: http://www.shallalist.de. [Accessed: 17-Apr-2018].

[34] A. C. Squicciarini, G. Petracca, W. G. Horne, and A. Nath, "Situational awareness through reasoning on network incidents," Proceedings of the 4th ACM conference on Data and application security and privacy CODASPY 14, 2014 https://doi.org/10.1145/2557547.2557562

[35] K. Jazayeri, M. Jazayeri, and S. Uysal, "Comparative Analysis of Levenberg-Marquardt and Bayesian Regularization Backpropagation Algorithms in Photovoltaic Power Estimation Using Artificial Neural Network," Advances in Data Mining. Applications and Theoretical Aspects Lecture Notes in Computer Science, pp. 80-95, 2016. https://doi.org/10.1007/978-3-319-41561-1_7

[36] F. Dalipi and S. Y. Yayilgan, "The impact of environmental factors to skiing injuries: Bayesian regularization neural network model for predicting skiing injuries," 2015 6th International Conference on Computing, Communication and Networking Technologies (ICCCNT), 2015. https://doi.org/10.1109/ICCCNT.2015.7395218 